\title{
A micro-mechanically based quadratic yield condition for textured polycrystals
}

\author{
A. Bertram ${ }^{1}$, T. Böhlke ${ }^{2}$, G. Risy ${ }^{3}$
}

\author{
${ }^{1}$ Universität Magdeburg, Institut für Mechanik, Postfach 4120, D-39016 Magdeburg, Germany \\ URL: www.uni-magdeburg.de/ifme/festigkeit.html, e-mail: bertram@mb.uni-magdeburg.de \\ ${ }^{2}$ Universität Karlsruhe (TH), Institut für Technische Mechanik, Postfach 6980, D-76128 Karlsruhe, Germany \\ URL: www.itm-uni-karlsruhe.de e-mail: boehlke@itm.uni-karlsruhe.de; \\ ${ }^{3}$ Universität Magdeburg, Institut für Mechanik, Postfach 4120, D-39016 Magdeburg, Germany \\ URL: www.uni-magdeburg.de/ifme/festigkeit.html, e-mail: risy@mb.uni-magdeburg.de
}

\begin{abstract}
In the present paper a two-scale approach for the description of anisotropies in sheet metals is introduced, which combines the advantages of a macroscopic and a microscopic modeling. While the elastic law, the flow rule, and the hardening rule are formulated on the macroscale, the anisotropy is taken into account in terms of a micro-mechanically defined 4th-order texture coefficient. The texture coefficient specifies the anisotropic part of the elasticity tensor and the quadratic yield condition. The evolution of the texture coefficient is described by a rigid-viscoplastic Taylor type model. The advantage of the suggested model compared to the classical v. Mises-Hill model is first that macroscopic anisotropy parameters can be identified based on a texture measurement, and second that the anisotropy of the elastic and the plastic behavior is generally pathdependent, and that this path-dependence is related to a micro-mechanical deformation mechanism. An explicit modeling of the plastic spin is circumvented by the aforementioned micro-mechanical approach. The model is implemented into the FE code ABAQUS and applied to the simulation of the deep drawing process of aluminum.
\end{abstract}

KEYWORDS: crystallographic texture, deformation-induced anisotropy, macroscopic yield condition, metal forming, polycrystalline material

\section{INTRODUCTION}

The deep drawing process of metals is often simulated by the application of the finite element method in combination with a phenomenological anisotropic elastic-plastic material model (see, e.g. Barlat et al., 1997, 2005). The main advantage of phenomenological approaches are the relatively low computational costs. The main disadvantage, however, is that the evolution of the anisotropy during the deformation process is neglected.

In contrast to phenomenological approaches, polycrystal plasticity models allow for a description of an evolving microstructure. Since such models are based on constitutive equations on the crystalline level, they take into account micro-mechanical deformation mechanisms (see, e.g. Bronkhorst et al., 1992). Although the models are relatively accurate, they have the disadvantage that large scale simulations are very time consuming.
Several authors have discussed how the numerical effort could be reduced when the stress is computed based on crystal plasticity models at the integration points. These approaches use an artificial scattering of the crystal orientations from integration point to integration point (Raabe and Roters, 2004), or the modeling of an isotropic background of the texture (Böhlke et al., 2006), or the determination of optimal sets of discrete crystal orientations (Schulze, 2006).

In the present paper, a model approach is suggested which combines the advantages of both a macroscopic and a microscopic approach (Böhlke, 2005; Risy, 2007). While the elastic law, the flow rule, and the hardening rule are formulated with respect to the macroscale, a 4th-order texture coefficient is used to capture the macroscopic anisotropies. This texture coefficient is incorporated in the macroscopic elastic law and in the macroscopic flow rule. Its evolution is determined by the use of a rigidviscoplastic Taylor model. As a consequence, there 
is no need for an explicit modeling of the plastic spin. The rotation of the crystal lattice vectors in relation to the material is taken into account by the micromechanical model. The macroscopic anisotropy results from a specific orientation distribution on the microscale which changes with large inelastic deformations.

\section{Elastic law}

For the formulation of the geometrically nonlinear elastic-viscoplastic material model we start with the concept of materials with isomorphic elastic ranges (Bertram, 2005). This approach is closely related to the multiplicative decomposition of the deformation gradient $\boldsymbol{F}$. The internal variable is the plastic transformation $\boldsymbol{P}$, a path-dependent, unimodular, non-symmetric tensor. Based on the plastic transformation, we define

$$
\boldsymbol{F}_{e}=\boldsymbol{F P}
$$

which enters the elastic law.

The elastic strains are assumed to be small. Therefore, each linear relation between a conjugate pair of generalized stress and strain measures is applicable for the description of the elastic behavior. Here, we assume a linear relation between the 2nd-PiolaKirchhoff stress tensor and Green's strain tensor with respect to the undistorted state. In an Eulerian setting, this ansatz implies that the Kirchhoff stress tensor $\tau$ is given as a linear function of the Almansi strain tensor $\boldsymbol{E}_{e}^{A}$ (see, e.g., Böhlke and Bertram, 2001; Böhlke et al., 2003)

$$
\boldsymbol{\tau}=\mathbb{C}_{e}\left[\boldsymbol{E}_{e}^{A}\right], \boldsymbol{E}_{e}^{A}=\frac{1}{2}\left(\boldsymbol{I}-\boldsymbol{B}_{e}^{-1}\right), \boldsymbol{B}_{e}=\boldsymbol{F}_{e} \boldsymbol{F}_{e}^{\top}
$$

with $I$ being the unit tensor. The Kirchhoff stress tensor $\boldsymbol{\tau}=J \boldsymbol{\sigma}$ is defined by the Cauchy stress tensor $\boldsymbol{\sigma}$ and the determinant $J$ of $\boldsymbol{F}$. The Eulerian stiffness operator $\mathbb{C}_{e}$ is given by the Rayleigh product of $\boldsymbol{F}_{e}$ and the reference stiffness tensor $\widetilde{\mathbb{C}}$ (see, e.g., Bertram, 2005)

$$
\begin{aligned}
& \mathbb{C}_{e}=\boldsymbol{F}_{e} \star \tilde{\mathbb{C}}= \\
& \tilde{C}_{i j k l}\left(\boldsymbol{F}_{e} \boldsymbol{e}_{i}\right) \otimes\left(\boldsymbol{F}_{e} \boldsymbol{e}_{j}\right) \otimes\left(\boldsymbol{F}_{e} \boldsymbol{e}_{k}\right) \otimes\left(\boldsymbol{F}_{e} \boldsymbol{e}_{l}\right) .
\end{aligned}
$$

$\boldsymbol{e}_{i}$ denotes the fixed sample system.

For aggregates of cubic crystals, the Voigt bound and the Reuss bound can be represented as an additive split of the elasticity tensor into an isotropic and an anisotropic part (Böhlke and Bertram, 2001; Böhlke et al., 2003). Here, we apply such a split to the effective elasticity tensor

$$
\tilde{\mathbb{C}}=\tilde{\mathbb{C}}^{I}+\tilde{\mathbb{C}}^{A} .
$$

Using the polar decomposition $\boldsymbol{F}_{e}=\boldsymbol{R}_{e} \boldsymbol{U}_{e}$ and considering small elastic strains, e.g. $\boldsymbol{U}_{e} \approx \boldsymbol{I}$, the following approximation for the Eulerian stiffness tensor is obtained

$$
\mathbb{C}_{e} \approx \tilde{\mathbb{C}}^{I}+\mathbb{C}_{e}^{A} .
$$

The isotropic part $\tilde{\mathbb{C}}^{I}$ has the following representation

$$
\tilde{\mathbb{C}}^{I}=3 K \mathbb{P}_{1}^{I}+2 G \mathbb{P}_{2}^{I}
$$

$K$ is the bulk modulus and $G$ is the shear modulus. The tensors $\mathbb{P}_{1}^{I}$ and $\mathbb{P}_{2}^{I}$ are the isotropic projectors

$$
\mathbb{P}_{1}^{I}=\frac{1}{3} \boldsymbol{I} \otimes \boldsymbol{I}, \quad \mathbb{P}_{2}^{I}=\mathbb{I}^{S}-\mathbb{P}_{1}^{I} .
$$

$\mathbb{I}^{S}$ is the identity tensor on symmetric 2 nd-order tensors. $\tilde{\mathbb{C}}^{I}$ is assumed to be constant during the deformation process.

If we neglect the lattice distortions, which is an assumption reasonable for small elastic strains, then the anisotropic part of the stiffness tensor $\mathbb{C}_{e}^{A}$ can be described in terms of the 4th-order texture coefficient $\mathbb{V}^{\prime}$ (Böhlke, 2005, 2006)

$$
\mathbb{C}_{e}^{A}=\zeta \mathbb{V}^{\prime}
$$

$\zeta$ depends on the eigenvalues of the single crystal stiffness tensor. In Böhlke et al. (2003) the evolution of the tensor $\mathbb{V}^{\prime}$ during the deformation process is modeled by a macroscopic constitutive equation. Here, this tensor is calculated based on a discrete orientation distribution. For a set of $N$ crystal orientations and corresponding volume fractions $\left\{\boldsymbol{Q}_{\alpha}, \nu_{\alpha}\right\}$, the tensor $\mathbb{V}^{\prime}$ is given by

$$
\mathbb{V}^{\prime}=\frac{3 \sqrt{30}}{10}\left(5 \sum_{\alpha=1}^{N} \nu_{\alpha} \boldsymbol{Q}_{\alpha} \star \sum_{i=1}^{3} \mathbb{D}_{0}-\boldsymbol{I} \otimes \boldsymbol{I}-2 \mathbb{I}\right)
$$

with

$$
\mathbb{D}_{0}=\boldsymbol{e}_{i} \otimes \boldsymbol{e}_{i} \otimes \boldsymbol{e}_{i} \otimes \boldsymbol{e}_{i}
$$

(Böhlke, 2005, 2006). In the last equation, the orthogonal tensor $\boldsymbol{Q}_{\alpha}$ represents the orientation $\boldsymbol{Q}$ of the $\alpha$-th crystal. 


\subsection{Flow rule}

For elastically anisotropic materials a ninedimensional flow rule is generally required. The key contribution of this paper is the separation of the plastic spin and the evolution of the elastic strain. This separation is obtained as follows. Since $\mathbb{V}^{\prime}$ is calculated based on a micro-mechanical model, the amount and type of anisotropy and the anisotropy directions can be determined based on the orientation distribution. Hence for given orientation distribution, the Eulerian stiffness tensor $\mathbb{C}_{e}$ is known. For the determination the stress $\boldsymbol{\tau}$ beside $\mathbb{C}_{e}$ only $\boldsymbol{B}_{e}$ has to be determined. Therefore, only a symmetric, i.e., sixdimensional flow rule has to be specified here. The material time derivative of $\boldsymbol{B}_{e}$ is

$$
\dot{\boldsymbol{B}}_{e}=\dot{\boldsymbol{F}}_{e} \boldsymbol{F}_{e}^{\top}+\boldsymbol{F}_{e} \dot{\boldsymbol{F}}_{e}^{\top} .
$$

The rate of change of the plastic transformation is assumed to depend on the stress state, the hardening state, and the crystallographic texture by means of the 4th-order texture coefficient

$$
\boldsymbol{P}^{-1} \dot{\boldsymbol{P}}=-\dot{\boldsymbol{F}}_{p} \boldsymbol{F}_{p}^{-1}=-\tilde{\boldsymbol{k}}\left(\boldsymbol{T}_{e}^{\prime}, \tilde{\mathbb{V}}^{\prime}, \sigma_{F}\right) .
$$

$\sigma_{F}$ is the macroscopic flow stress, $\tilde{\mathbb{V}}^{\prime}=\boldsymbol{F}_{e}^{-1} \star \mathbb{V}^{\prime}$ is the texture coefficient pulled back to the undistorted configuration, $\boldsymbol{T}_{e}^{\prime}$ is the Mandel stress tensor given by $\boldsymbol{T}_{e}=\boldsymbol{C}_{e} \boldsymbol{S}_{e}$ with the 2nd Piola-Kirchhoff stress tensor $\boldsymbol{S}_{e}$ and the right Cauchy-Green tensor $\boldsymbol{C}_{e}=\boldsymbol{F}_{e}^{\top} \boldsymbol{F}_{e}$. Combining (11) and (12) we find

$$
\begin{aligned}
\mathcal{L}\left(\boldsymbol{B}_{e}\right) & =\dot{\boldsymbol{B}}_{e}-\boldsymbol{L} \boldsymbol{B}_{e}-\boldsymbol{B}_{e} \boldsymbol{L}^{\top} \\
& =-2 \operatorname{sym}\left(\boldsymbol{k}_{e}\left(\boldsymbol{\tau}^{\prime}, \mathbb{V}^{\prime}, \sigma_{F}\right) \boldsymbol{B}_{e}\right)
\end{aligned}
$$

with

$$
\boldsymbol{k}_{e}\left(\boldsymbol{\tau}^{\prime}, \mathbb{V}^{\prime}, \sigma_{F}\right)=\boldsymbol{F}_{e} \tilde{\boldsymbol{k}}\left(\boldsymbol{T}_{e}^{\prime}, \mathbb{V}^{\prime}, \sigma_{F}\right) \boldsymbol{F}_{e}^{-1}
$$

$\boldsymbol{L}$ is the velocity gradient. We assume the existence of an Eulerian flow potential $\phi\left(\boldsymbol{\tau}^{\prime}, \mathbb{V}^{\prime}, \sigma_{F}\right)$ such that

$$
\boldsymbol{k}_{e}\left(\boldsymbol{\tau}^{\prime}, \mathbb{V}^{\prime}, \sigma_{F}\right)=\frac{\partial \phi\left(\boldsymbol{\tau}^{\prime}, \mathbb{V}^{\prime}, \sigma_{F}\right)}{\partial \boldsymbol{\tau}^{\prime}}
$$

holds. A common form of the flow potential in the context of viscoplasticity is given by

$$
\phi=\frac{\dot{\varepsilon}_{0} \sigma_{F}}{m+1}\left(\frac{\sigma_{e q}}{\sigma_{F}}\right)^{m+1} .
$$

$m$ and $\dot{\varepsilon}_{0}$ are material parameters. In order to incorporate the texture coefficient, we formulate the equivalent stress in terms of an anisotropic norm (Böhlke, 2005)

$$
\sigma_{e q}\left(\boldsymbol{\tau}^{\prime}, \mathbb{V}^{\prime}\right)=\sqrt{\frac{3}{2}}\left\|\boldsymbol{\tau}^{\prime}\right\|_{\mathbb{H}}=\sqrt{\frac{3}{2}} \sqrt{\boldsymbol{\tau}^{\prime} \cdot \mathbb{H}\left[\boldsymbol{\tau}^{\prime}\right]}
$$

with

$$
\mathbb{H}=\mathbb{P}_{2}^{I}+\eta \mathbb{V}^{\prime} .
$$

The parameter $\eta$ has to be chosen such that the tensor $\mathbb{H}$ is positive definite on the set of traceless and symmetric tensors. It should be noted that in the rate-independent limit, i.e. $m \rightarrow \infty$, the classical quadratic yield condition by v. Mises (1928) and Hill (1948) is obtained

$$
\sqrt{\boldsymbol{\tau}^{\prime} \cdot \mathbb{H}\left[\boldsymbol{\tau}^{\prime}\right]}-\sqrt{\frac{2}{3}} \sigma_{F}=0 .
$$

Finally, we derive the following form for $\boldsymbol{k}_{e}$

$$
\boldsymbol{k}_{e}\left(\boldsymbol{\tau}^{\prime}, \mathbb{V}^{\prime}, \sigma_{F}\right)=\frac{3}{2} \frac{\dot{\varepsilon}_{0}}{\sigma_{e q}}\left(\frac{\sigma_{e q}}{\sigma_{F}}\right)^{m}\left(\mathbb{P}_{2}^{I}+\eta \mathbb{V}^{\prime}\right)\left[\boldsymbol{\tau}^{\prime}\right]
$$

It can be seen that the texture coefficient governs the flow direction. In the isotropic case, i.e. $\mathbb{V}^{\prime}=0$, the last equation reduces to the isotropic v. Mises flow rule.

\section{CONCLUSIONS}

In the present paper a two-scale approach has been suggested in order to simulate the mechanical behavior of polycrystals under large plastic deformations. This approach is based on constitutive equations which are formulated with respect to the macroscale containing, micro-mechanically defined internal variables. The evolution of the micromechanical variable has been taken into account based on a Taylor type model. Generally, both the evolving elastic and plastic anisotropies can be modeled by the suggested approach.

The simulated earing profiles reproduce the features of the experimental findings. Compared to classical Taylor type models, the computation of the macroscopic stress is much simpler and faster. Since the texture evolves slowly compared to the yield stress, an update of the texture coefficient is not required in each time step. Furthermore, even if only 
a small number of crystal orientations is used, the anisotropy is not necessarily overestimated since the discrete orientations enter the model through the 4thorder texture coefficient specifying the quadratic flow rule.

\section{REFERENCES}

ABAQUS/Standard (2007). Hibbitt, Karlsson \& Sorensen, Inc.

Barlat, F., Becker, R., Hayashida, Y., Maeda, Y., Yanagawa, M., Chung, K., Brem, J., Lege, D., Matsui, K., Murtha, S., and Hattori, S. (1997). Yielding description for solution strengthened aluminum alloys. Int. J. Plast., 13(4), 385-401.

Barlat, F., Aretz, H., Yoon, J., Karabin, M., Brem, J., and Dick, R. (2005). Linear transformation-based anisotropic yield functions. Int. J. Plast., 21(4), 1009-1039.

Bertram, A. (2005). Elasticity and Plasticity of Large Deformations. Springer-Verlag, Berlin.

Böhlke, T. and Bertram, A. (2001). The evolution of Hooke's law due to texture development in polycrystals. Int. J. Solids Struct., 38(52), 9437-9459.

Böhlke, T., Bertram, A., and Krempl, E. (2003). Modeling of deformation induced anisotropy in free-end torsion. Int. J. Plast., 19, 1867-1884.

Böhlke, T. (2005). Two-scale modeling of plastic anisotropies. Proceedings of the VIII International Conference on Computational Plasticity, COMPLAS VIII, E. Onate and D.R.J. Owen (Eds), 610613.

Böhlke, T. (2005). Application of the maximum entropy method in texture analysis. Comp. Mat. Science, 32, 276-283.

Böhlke, T. (2006). Texture simulation based on tensorial Fourier coefficients. Computers and Structures, 84, 1086-1094.
Böhlke, T., Risy, G., and Bertram, A. (2006). Finite element simulation of metal forming operations with texture based material models. Modelling Simul. Mater. Sci. Eng., 14, 1-23.

Bronkhorst, C., Kalidindi, S., and Anand, L. (1992). Polycrystalline plasticity and the evolution of crystallographic texture in fcc metals. R. Soc. Lond., A341, 443-477.

Bunge, H.-J. (1993). Texture Analysis in Material Science. Cuviller Verlag Göttingen.

Hill, R. (1948). A theory of yielding and plastic flow of anisotropic materials. Proc. Phys. Soc. Lond., A 193, 281-297.

Mandel, J. (1974). Thermodynamics and plasticity. In J. Delgado Domingos, M. Nina, and J. Whitlaw, Eds, Proc. Int. Symp. Foundations of Continuum Thermodynamics, McMillan, London, 283.

Raabe, D. and Roters, F. (2004). Using texture components in crystal plasticity finite element simulations. Int. J. Plast, 20, 339-361.

Risy, G. (2007). Modellierung der texturinduzierten plastischen Anisotropie auf verschiedenen Skalen. Ph.D. thesis, Otto von Guericke Universität Magdeburg, http://diglib.unimagdeburg.de/verzeich/fmb.htm.

Schulze, V. (2006). Anwendung eines kristallplastischen Materialmodells in der Umformsimulation. Ph.D. thesis, Otto von Guericke Universität Magdeburg, http://diglib.unimagdeburg.de/verzeich/fmb.htm.

Taylor, G. (1938). Plastic strain in metals. J. Inst. Metals, 62, 307-324.

v. Mises, R. (1928). Mechanik der plastischen Formänderung bei Kristallen. Z. angew. Math. Mech., 8 (3), 161-185. 